\title{
Synchrotron Radiation for the Understanding of Block Copolymer Self-assembly
}

\author{
Marta Fernández-Regúlez , Christian Pinto-Gómez, and Francesc Perez-Murano \\ Instituto de Microelectrónica de Barcelona, IMB-CNM (CSIC), \\ Campus UAB, 08193 Bellaterra, Spain \\ *marta.fernandez@imb-cnm.csic.es
}

\begin{abstract}
Synchrotron radiation offers great opportunities to improve the understanding of block copolymer morphology and self-assembly processes. In particular, GISAXS characterization is an interesting complementary method to analyze with sub-nanometer resolution the patterns that block copolymer thin films form, in addition to chemical composition. Most of the synchrotron radiation techniques are compatible with block copolymer processes, allowing for an in-situ and real time analysis of the self-assembly processes, what is very important for the analysis of process kinetics. In this contribution, we present the analysis of the self-assembly process of lamellar PS-b-PMMA block copolymer submitted to thermal annealing. The analysis of the 2D-GISAXS patterns allows the evaluation of the block copolymer morphology on formed nanopatterns and about the block copolymer kinetics.
\end{abstract}

Keywords: Block copolymer, metrology, synchrotron, GISAXS

\section{Introduction}

During the last years, block copolymer based manufacturing has demonstrated its potential as a patterning method thanks to the achieved high resolution, up to few nanometers, its process low cost and simplicity [1]. In particular, the directed self-assembly (DSA) of block copolymer has been proposed as a complementary technique for high volume manufacturing for the semiconductor industry [2].

Block copolymer are macromolecules composed of two or more homo-polymer chains, or blocks, joined together through a covalent bond. Due to this special configuration, when segregation strength between blocks is strong enough they tend to undergo a phase separation on periodic structures such as lamellas, cylinders or spheres [3]. Standard top-down lithography can be used to fabricate chemical (chemoepitaxy) [4,5] or topographical guiding patterns (graphoepitaxy) [6] to organize and direct their self-assembly on predefined wellordered features. DSA of block copolymer is not a lithography technique by itself but it is considered a complementary technique to improve the resolution, throughput and roughness of conventional standard top-down approaches.
However, the full integration of DSA processes on semiconductor industry is still under evaluation. There are still material, and engineering challenges that must be fulfilled [7]. In this scenario, appropriate metrology techniques are crucial for the study of process parameters such as annealing conditions, defectivity level and pattern line-edge roughness. The characterization is usually performed using real-space techniques such as atomic force microscopy (AFM) or scanning electron microscopy techniques (SEM). However, the information given by these techniques is restricted to a small inspection area and it only arises from the top layer surface, while many defects remain hidden through the block copolymer film. A more deep 3D-characterization of the block copolymer layer has been achieved by using sequential infiltration synthesis of alumina of one of the block and transmission electron microscopy (TEM) for the structural imaging [8]. However, in this case, sample preparation is very complex and the analysis is restricted to small areas and to block copolymers with a particular chemistry where one of the blocks can be selectively infiltrated.

Additionally, beside the characterization of static nanostructured block copolymer layers, the study of 
self-assembly kinetic is also relevant for the understanding and optimization of DSA processes [9]. Modelling of block copolymer self-assembly can be used to predict the kinetics. There are different studies about the evolution of defects on block copolymer film under different self-assembly conditions [10], but the information normally extracted from already self-assembled layers instead of the in-situ observation of the film evolution [11]. Then, the characteristic parameter of block copolymer order, the correlation length, is evaluated from SEM or AFM images of the samples that have been annealed at different conditions after being cold down [12]. The in-situ and real time observation of block copolymer thin film evolution using standard microscopy techniques becomes more complicated due to the process incompatibility with self-assembly process (high temperature or solvent atmosphere). Additionally, they are usually not fast enough to resolved the kinetics for short periods of time and the analyzed area per image is not representative enough for a statistical study.

Synchrotron radiation offers great possibilities to complement standard real-space characterization of block copolymer morphology and self-assembly characteristics. The information is collected from a relatively large volume due to the large area exposed by the synchrotron beam and the penetration depth of the beam. Synchrotron techniques such as High Kinetics Energy Photoelectron Spectroscopy (HAXPES) and Grazing Incident Small Angle Spectroscopy (GISAXS) have already demonstrated their potential for the study of block copolymer processes. In the first one, HAXPES has been used to investigate surface chemical changes that occurs during processing steps in DSA [13]. Using synchrotron radiation in HAXPEX, the penetration depth of the photons was tuned to extract information of dominant interactions between a random copolymer brush layer and the block copolymer by adjusting their energy (and thus kinetic energy).

On the other hand, Grazing Incident Small Angle $\mathrm{X}$-ray Spectroscopy (GISAXS) is also a powerful technique for the analysis of block copolymer thin films [14]. The 2D-GISAXS pattern gives information about the order and the shape of the nanostructured block copolymer layer and the analyzed area is very large due to the dimensions of the beam (between hundreds of square microns and square millimeters) and the small incident angle.
GISAXS characterization has been used for the characterization of DSA patterns. Patterns created by graphoepitaxy of a PS-b-PMMA cylindrical block copolymer has been analyzed using GISAXS showing a typical GISAXS of a single domain order for optimal self-assembly conditions and guiding pattern commensurability [15]. Multiple processing parameters such as PMMA removal, film thickness and annealing treatment were evaluated by ex-situ GISAXS characterization [16]. Recently, we have presented the analysis of nanopatterns created by directing the self-assembly of the lamellar PS- $b$ PMMA block copolymer on sub-10 nm topographical guiding patterns [17]. Block copolymer DSA on different guiding structures were inspected but the ones with optimal dimensions showed a higher intensity on the interference 2D-GISAXS pattern.

The characterization of thin films using GISAXS is also compatible with standard self-assembly condition (thermal or solvent annealing) that, together with the highly time-resolved data acquisition, makes this technique ideal for in-situ and real-time characterization of self-assembly process. However, the majority of the studies using GISAXS on block copolymer thin films have been performed in static mode on previously selfassembled layers and most of the studies of in-situ real-time block copolymer evolution are mainly related to solvent annealing process $[18,19]$

In this paper, we present the in-situ and real time characterization of the self-assembly process of a lamellar PS-b-PMMA Poly[styrene-block-(methyl methacrylate)]. The block copolymer will be annealed under isothermal conditions and the parameters of the block copolymer kinetics will be extracted from 2D-GISAXS patterns taken during the annealing process.

\section{Methodology}

2.1. Block copolymer self-assembly

Block copolymer was deposited on $2 \times 2 \mathrm{~cm}$ silicon samples. First, in order to promote the perpendicular alignment of the lamellas, the surface was conditioned with a brush of Poly (styreneramdom-methylmetacrylate), PS-r-PMMA, with neutral affinity for both polymer blocks. The weight $M_{\text {brush }}=7.9 \mathrm{~kg} \mathrm{~mol}^{-1}(58 \mathrm{wt} \%$ PS and $42 \mathrm{wt} \%$ PMMA) and the polydispersity index $\mathrm{PDI}_{\text {brush }}=1.85$. The PS- $r$-PMMA polymer was diluted at $1.5 \mathrm{wt} \%$ in PGMEA (Propylene glycol methyl ether acetate) solvent and deposited by spin-coating at $2000 \mathrm{rpm}$. A thermal annealing at $230{ }^{\circ} \mathrm{C}$ for $5 \mathrm{~min}$ was used 
for the grafting and the remaining un-grafted polymer was removed by rinsing on PGMEA.

The block copolymer was diluted at $1.5 \mathrm{wt} \%$ in PGMEA and deposited by spin-coating. Samples were spin-coated on the bush coated samples at $2500 \mathrm{rpm}$ and immediately placed at the GISAXS sample holder to perform the annealing at different conditions. The block copolymer used for the experiments was PS- $b$-PMMA di-block copolymer with a lamellar morphology (molecular weight $M_{\mathrm{PS}-}$ b-PMMA $=42.3 \mathrm{~kg} \mathrm{~mol}^{-1}, 43 \mathrm{wt} \%$ of PS, $57 \mathrm{wt} \%$ PMMA, $\mathrm{PDI}_{\mathrm{BCP}}=1.1$ ). The natural period in free surface is $23.4 \mathrm{~nm}$.

\subsection{Sample analysis}

GISAXS characterization was conducted at the Austrian beamline at Elettra in Italy. The energy of the beam was $8.05 \mathrm{keV}$, which corresponds to a wavelength of $0.156 \mathrm{~nm}$. The incident angle was $0.15^{\circ}$ and the sample detector distance was $1.877 \mathrm{~m}$ for all the measurements. The detector was a Pilatus $31 \mathrm{M}$ system with a readout time of $0.95 \mathrm{~ms}$ and a pixel size of $172 \times 172 \mu \mathrm{m}$.
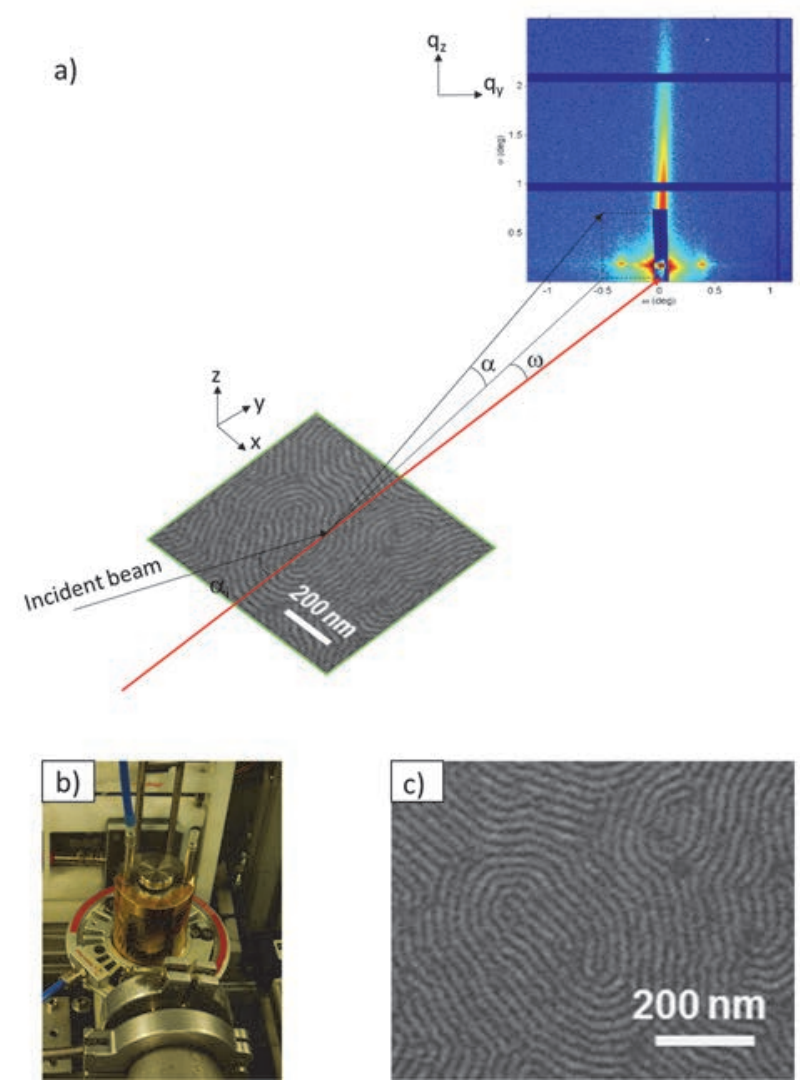

Fig. 1. a) Geometry of the GISAXS experiments, b) Sample holder for GISAXS characterization, c) SEM image of the self-assembled PS-b-PMMA block copolymer (the period is $23.4 \mathrm{~nm}$ ).
A Peltier heating/cooling sample holder (Aton Paar, Graz, Austrian) was used for the annealing of the samples (Fig. 1b). A small cylindrical chamber with kapton windows was used to keep the sample under nitrogen atmosphere during the experiments. The sample was placed at room temperature at the holder and then the temperature was heated up to the target annealing temperature. The heating rate was $50{ }^{\circ} \mathrm{C} / \mathrm{min}$.

In order to avoid beam damage on the samples, the acquisition time per GISAXS patterns was reduced to $1 \mathrm{~s}$ and the number of images per sample was limited to 20 frames.

\section{Results and discussions}

A characteristic GISAXS pattern of vertically oriented lamellar block copolymer sample presents Bragg rods along the $q_{\mathrm{y}}$ direction. The lamellar spacing, or period, can be determined from the position, $q_{\mathrm{y}}$, of the first order rod according the relation $L_{0}=2 \pi / q_{\mathrm{y}}$, while the correlation length is related with their width. As block copolymer is being ordered during the self-assembly process, rods become narrower. The correlation length is calculated from the width of the first order Bragg peak as Eq. 1:

$$
\xi=\frac{2 \pi k}{f w h m}
$$

where the "fwhm" is full width half-maximum of the first order Bragg rod and $\mathrm{k}$ is a correction parameter related to the detector type and the fitting of the peak. For the detector used in the experiments, an area detector, and a Gaussian fitting $k$ is 0.96 [20].

\subsection{Block copolymer heating ramp}

In Fig. 2, we can observe the GISAXS patterns during the heating ramp to reach the annealing temperature. The sample was placed at the GISAXS holder at room temperature and then the temperature was increased up to $225^{\circ} \mathrm{C}$ at a heating rate of $50{ }^{\circ} \mathrm{C} / \mathrm{min}$. After block copolymer spincoating and when sample is still at low temperature, layer is in amorphous state and periodicity is not observe on the patterns. At $156^{\circ} \mathrm{C}$, block copolymer layer starts to re-arrange and Bragg rods start to appear on the GISAXS pattern. This evolution is clear when we observe the evolution of the Horizontal projection at Yoneda band (Fig. 2). 

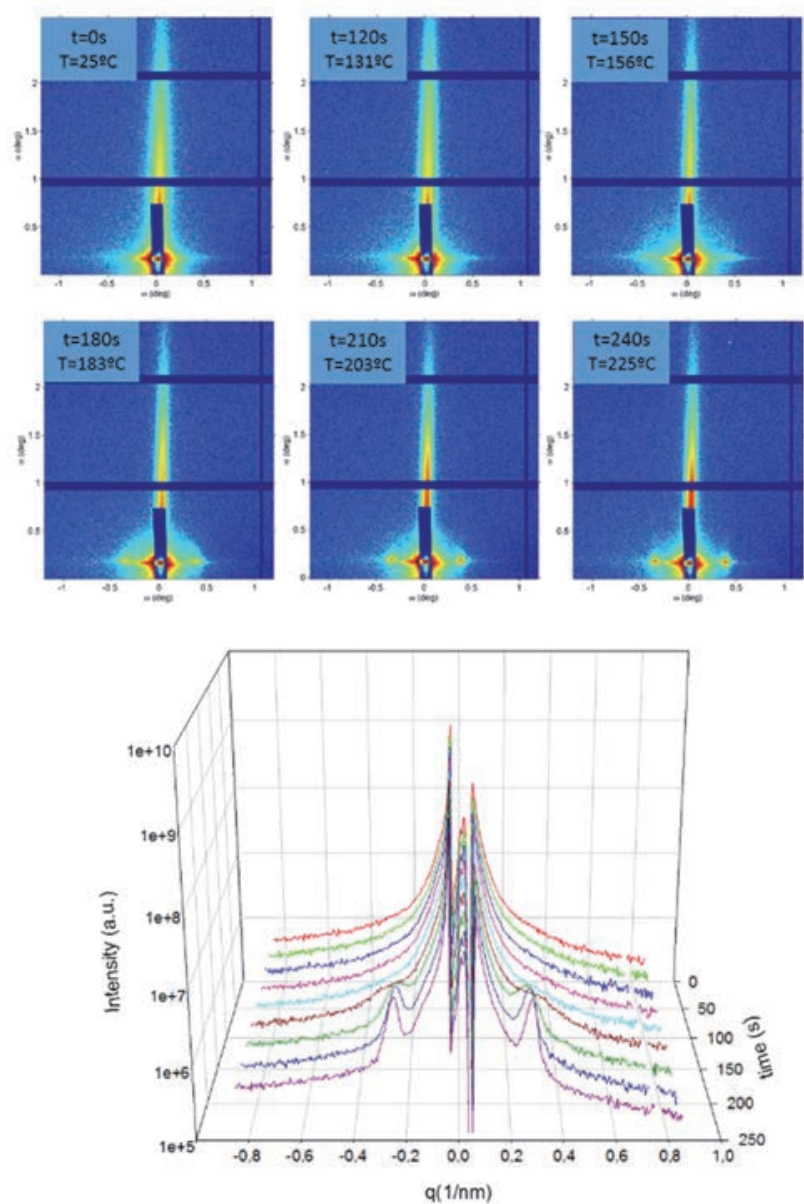

Fig. 2. Evolution of block copolymer GISAXS pattern during the heating ramp (top). GISAXS pattern taken during the heating ramp at $50{ }^{\circ} \mathrm{C} / \mathrm{min}$ (bottom). Evolution of the Horizontal cut at Yoneda band. For the estimation of the horizontal profile, 10 horizontal detector pixels are integrated.

\subsection{Block copolymer isothermal annealing}

Under isothermal annealing, the evolution of the correlation length of a block copolymer film is governed by a power law [21] as shown in Eq. 2:

$$
\xi=A_{T} t^{\phi}
$$

The equation is valid for a time $(t>t 0)$ at which the self-assembling of the block copolymer starts. $\phi$ (growth exponent that depends of the polymer and annealing conditions) has usually a value around $0.3-0.1$. The parameter $A_{T}$ is known to be dependent of the temperature having an Arrhenius behavior, $A_{T}=A_{0} e^{-E_{A} / R T}$, where $E_{\mathrm{A}}$ is the activation energy of the block copolymer dependent of the polymer chemistry and composition.

For self-assembly studies, block copolymer samples are placed at the holder and heated-up to the target self-assembly temperature. Once the annealing temperature is reached, the sample is kept under isothermal annealing for several minutes. A GISAXS image was taken every 60 seconds during the annealing to study de evolution of block copolymer thin film.

a)

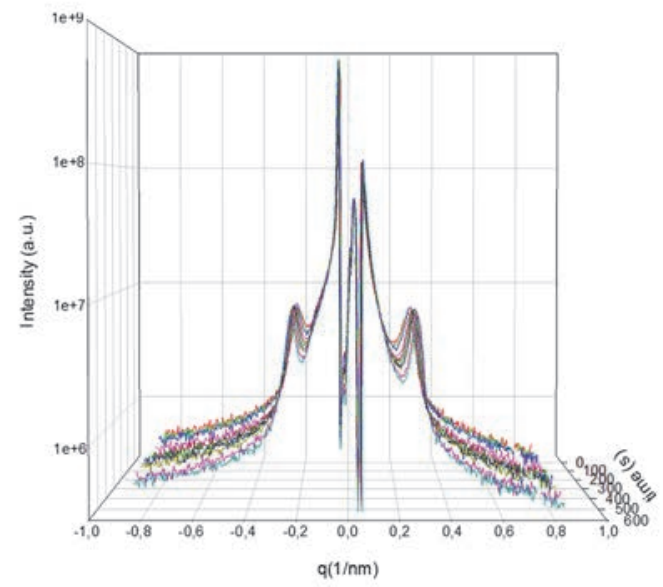

b)

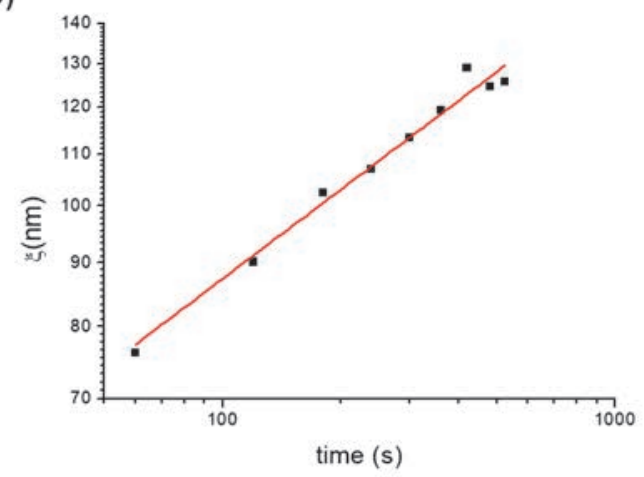

Fig. 3. Evaluation of block copolymer isothermal annealing at $170{ }^{\circ} \mathrm{C}$. (a) Evolution of the horizontal profile at lobe position (integration of 10 vertical pixels). b) Correlation length of the block copolymer as a function of the annealing time.

In Fig. 3, we can observe the evolution of the block copolymer film during an isothermal annealing at $170{ }^{\circ} \mathrm{C}$. For the estimation of the correlation length, horizontal profiles have been extracted from the 2D-GISAXS patterns at the lobe position and Gaussian fitting has been performed on the first order Bragg rod. In Fig. 3b, it can be observed the evolution of the correlation length as a function of the annealing time. This evolution follows the expected power low with a growth exponent $\phi=0.237$.

\section{Conclusion}

The characterization of block copolymer selfassembly morphology and process kinetics is of great interest for the semiconductor industry in 
order to evaluate their suitability for next generation technological nodes. Standard characterization techniques in real space are used for their characterization but they do not offer enough statistical data and are not compatible with the characterization of block copolymer kinetics at real time. Here, we have showed how synchrotron radiation and in particular GISAXS can complement the study of block copolymer. In particular, we have demonstrated the characterization of parameters governing the selfassembly process of a lamellar PS- $b$-PMMA block copolymer. We have also showed how the evolution of self-assembled layer and, in particular, the correlation length, successfully fulfil a power law governing in this kind of systems.

\section{Acknowledgements}

This work was partly supported by projects Nanointegra (TEC2015-69864-R), project NFFA (Nanoscience Foundries and Fine Analysis), and 2017-SGR-1187 (NANONEMS). We gratefully acknowledge ARKEMA for supplying us with the random and block copolymer materials.

\section{References}

1. H. Feng, X. Lu, W. Wang, N. G. Kang, and J. W. Mays, Polymers, 9 (2017) 494.

2. S. J. Jeong, J. Y. Kim, B. H. Kim, H. S. Moon, and S. O. Kim, Mater. Today, 16 (2013) 468.

3. F. Bates, Annu. Rev. Phys. Chem., 41 (2002) 525.

4. M. Fernandez-Regulez, L. Evangelio, M. Lorenzoni, J. Fraxedas, and F. Perez-Murano, ACS Appl. Mater. Interfaces, 6 (2014) 21596.

5. L. Evangelio, M. Fernández-Regúlez, X. Borrisé, M. Lorenzoni, J. Fraxedas, and F. Pérez-Murano, J. Micro/Nanolith. MEMS MOEMS, 14 (2015) 033511.

6. S. Gottlieb, D. Kazazis, I. Mochi, L. Evangelio, M. Fernández-Regúlez, Y. Ekinci, and F. PerezMurano, Soft Matter, 14 (2018) 6799.

7. C. M. Bates, M. J. Maher, D. W. Janes, C. J. Ellison, and C. G. Willson, Macromolecules, 47 (2014) 2.

8. T. Segal-Peretz, J. Ren, S. Xiong, G. Khaira, A. Bowen, L. E. Ocola, R. Divan, M. Doxastakis, N.
J. Ferrier, J. de Pablo, and P. F. Nealey, ACS Nano, 11 (2017) 1307.

9. W. Li and M. Müller, Annu. Rev. Chem. Biomol. Eng., 6 (2015) 187.

10.M. Müller, W. Li, J. C. O. Rey, and U. Welling, J. Phys. Conf. Ser., 640 (2015) 1.

11.C. T. Black, C. Forrey, and K. G. Yager, Soft Matter, 13 (2017) 3275.

12.M. Perego, F. Ferrarese Lupi, M. Ceresoli, T. J. Giammaria, G. Seguini, E. Enrico, L. Boarino, D. Antonioli, V. Gianotti, K. Sparnacci, and M. Laus, J. Mater. Chem. C, 2 (2014) 6655.

13.L. Evangelio, F. Gramazio, M. Lorenzoni, M. Gorgoi, F. M. Espinosa, R. García, F. PérezMurano, and J. Fraxedas, Beilstein J. Nanotechnol., 8 (2017) 1972.

14.S. V. Roth, G. Santoro, J. F. H. Risch, S. Yu, M. Schwartzkopf, T. Boese, R. Döhrmann, P. Zhang, B. Besner, P. Bremer, D. Rukser, M. A. Rübhausen, N. J. Terrill, P. A. Staniec, Y. Yao, E. Metwalli, and P. Müller-Buschbaum, ACS Appl. Mater. Interfaces, 7 (2015) 12470.

15.M. Maret, R. Tiron, X. Chevalier, P. Gergaud, A. Gharbi, C. Lapeyre, J. Pradelles, V. Jousseaume, G. Fleury, G. Hadziioannou, N. Boudet, and C. Navarro, Macromolecules, 47 (2014) 7221.

16.G. Freychet, M. Maret, R. Tiron, X. Chevalier, A. Gharbi, M. Fernandez-Regulez, and P. Gergaud, J. Polym. Sci. Part B: Polym. Phys., 54 (2016) 1137.

17.S. Gottlieb, B. Rösner, L. Evangelio, M. Fernández-Regúlez, A. Nogales, M. C. GarcíaGutiérrez, T. F. Keller, J. Fraxedas, T. A. Ezquerra, C. David, and F. Perez-Murano, Mol. Syst. Des. Eng., (2018) 175.

18.D. Posselt, K. Kyriakos, C. M. Papadakis, D.-M. Smilgies, S. Jaksch, J. Perlich, and J. Zhang, Macromolecules, 47 (2014) 5711.

19.W. Bai, K. G. Yager, and C. A. Ross, Polymer, 101 (2016) 176.

20.D.-M. Smilgies, J. Appl. Crystallogr., 42 (2009) 1030.

21.D. A. Vega, C. K. Harrison, D. E. Angelescu, M. L. Trawick, D. A. Huse, P. M. Chaikin, and R. A. Register, Phys. Rev. E, 71 (2005) 61803. 